\title{
INTEGRANDO A IMPLANTAÇÃO DE TECNOLOGIAS DA INFORMAÇÃO APLICADAS À GESTÃO DA PRODUÇÃO COM ESTRATÉGIAS DE RECURSOS HUMANOS
}

\section{INTEGRATING THE IMPLEMENTATION OF INFORMATION TECHNOLOGY APPLICABLE TO OPERATIONS MANAGEMENT WITH HUMAN RESOURCE STRATEGIES}

\author{
José Manoel Souza das Neves'; Fernando César Almada Santos ${ }^{2}$ \\ ${ }^{1}$ FATEC/Centro Paula Souza, EESC/USP, jmneves@fatecguaratingueta.edu.br \\ ${ }^{2}$ EESC/USP, almada@producao.eesc.sc.usp.br
}

\author{
Recebido para publicação em: 11/02/2005 \\ Aceito para publicação em: 29/07/2005
}

\begin{abstract}
Resumo
$O$ artigo apresenta revisão bibliográfica e considerações sobre o processo de integração da implantação de tecnologias da informação aplicadas à gestão da produção com estratégias e práticas de gestão de recursos humanos. O uso de tecnologias da informação induz a novas formas de administração, exige um novo perfil do profissional de gestão com características diferentes das adotadas atualmente nas organizações, bem como implica no desenvolvimento de novos modelos de gestão de recursos humanos.
\end{abstract}

Palavras-chave: Tecnologia da informação, gestão de negócios, gestão de recursos humanos

\section{Introdução}

As organizações têm informatizado seus departamentos, adquirindo computadores, impressoras, instalando softwares e ligando-os em rede, acreditando ser suficiente para organizá-las (ABREU e RESENDE, 2001).

Vemos que os sistemas de informações empresariais estão gerando uma nova forma de vantagem competitiva. Face ao grande número de tecnologias que estão sendo introduzidas no mercado empresarial, a informação passa a ser um bem patrimonial tangível, capaz de garantir o desenvolvimento de uma empresa.

Drucker (1995) coloca que o mundo está migrando para uma sociedade do conhecimento devido a automação dos processos de trabalho. Nesta nova sociedade, a Tecnologia da Informação 
(TI) pode trazer um diferencial para as organizações que souberem utilizá-las de forma adequada, sendo um recurso para competir e permanecer no mercado.

Tem havido uma grande preocupação com a implementação efetiva da TI e seu impacto na organização. "A introdução de inovações deve ser planejada e administrada e o investimento associado deve estar em sintonia com as necessidades do mercado [...], bem como com seus objetivos e estratégias de curto, médio e longo prazo". O sucesso da adoção de TI está relacionado com o "saber escolher" e o "saber usar", o que pressupõe a assimilação de inovações tecnológicas, o alinhamento entre a TI e as estratégias da organização, a elaboração de estratégias específicas para investimentos em TI, bem como atitudes gerenciais e comportamentais voltadas para a inovação (FERNANDES e ALVES, 1992, p. 72).

A presença cada vez maior da tecnologia da informação nas empresas tem-se dado por razões estratégicas fundamentais: a competitividade crescente leva à necessidade de maior domínio sobre os parâmetros que estão em jogo, maior flexibilidade para adaptar-se a novas condições do mercado e maior capacidade de absorção de novas técnicas e tecnologias. (SPINOLA; PESSOA, 1997).

\section{Tecnologia da informação e a estratégia de negócio}

A tecnologia da informação reúne as contribuições da tecnologia e da administração para estabelecer uma estratégia integrada (negócios + organização + tecnologia), projetar e instalar sistemas de informação e as coerentes mudanças organizacionais. (WALTON, 1993)

Laurindo (2000, p.1) adota o conceito de Tecnologia da Informação (TI) formulado por Weil, que é mais amplo e inclui o uso de "hardware" e "software", telecomunicações, automação, recursos multimídia e todos os outros recursos e pessoal dedicados a TI, quer sejam centralizados ou descentralizados, sem deixar de considerar os sistemas de informação, serviços, negócios, usuários e as relações complexas envolvidas.

Para Walton (1993), a TI pode ser definida como a utilização das ferramentas de informática, comunicação e automação juntamente com as técnicas de organização e gestão alinhadas com a estratégia de negócio, com o objetivo de aumentar a competitividade da empresa.

Laudon e Laudon (1998) defendem que os sistemas de informações facilitam o fluxo dos dados, contribuindo para a produção do conhecimento aplicado ao trabalho e organização do conhecimento empresarial.

Além de envolver a empresa globalmente, a tecnologia da informação estabelece um caminho para sincronizar a implantação de novas tecnologias e as mudanças na estrutura e nas atividades da organização (WALTON, 1993) 
Martin et al. (1994) descreve que sistemas estratégicos de informação são um meio para implementação de estratégias que manipulam informações, transformando-as em conhecimento. $\mathrm{O}$ sistema permite também a comunicação da informação, transcendendo as fronteiras, focando os clientes, fornecedores e concorrentes. A estratégia pode ter um impacto abrangente sobre a empresa, mercado e indústria. Dessa forma, o sistema sustenta o processo de mudança organizacional e afirma "que a tecnologia da informação é importante por ser facilitadora destas transformações".

Para Laurindo (1995), a eficácia da tecnologia da informação depende de sua organização estrutural e da empresa podendo influenciar e mudar a estratégia e a administração do negócio.

Segundo Spinola e Pessoa apud Contador (1998), a informação é um elemento essencial para a organização, sendo integradora e alimentadora das atividades, podendo caricaturá-la como a "mola propulsora" da organização. A informação não é limitada a dados, mas todos os dados obtidos, organizados e úteis à organização, enquanto que o sistema de informação cria um ambiente facilitado, integrado e consistente. A Tecnologia da Informação é a aplicação da Tecnologia e dos princípios de Administração no contexto organizacional.

Furtado apud Contador (1998, p. 107) comentam que "a informação - que já foi considerada fator de produção - é reconhecida como recurso mais importante para a tomada de decisões, no completo ecossistema do homem econômico". No Brasil, as pequenas e médias empresas apresentam grande dificuldade de identificar suas necessidades e implementar as mudanças necessárias. O processo de logística enfrenta dificuldades no que se refere à introdução de novas tecnologias, tendo em vista a necessidade de integração de sistemas e processos.

Alcântara e Silva (2001) apontam alguns fatores críticos para a implementação bem sucedida de uma TI, no sentido de construir alianças estratégicas entre empresas e que podem ser divididas em características facilitadoras e limitadoras.

Entre as facilitadoras são apontadas:

- Existência de aderência e de sinergia estratégica e cultural, entre as empresas envolvidas;

- Dar um salto qualitativo em termos de estruturação de recursos humanos e investir em treinamento;

- Postura firme e definitiva da alta direção na incorporação dessas mudanças;

- Ter a expectativa de que haverá mudança no eixo de poder em algumas áreas da empresas e na distribuição de poder.

Como limitadoras são apontadas:

- Ausência de um modelo a ser seguido como sinalizador dessas mudanças que minimize os custos de erros decorrentes delas;

- Não existência de base conceitual comum, amplamente desenvolvida e entendida, que possa ser utilizada como linguagem comum; 
- Falta de recursos humanos capacitados para as novas tarefas;

- Resistência existente às mudanças necessárias, advinda de cultura organizacional estabelecida há vários anos e de toda a estrutura de poder gerada dentro das empresas.

Ferreira (1994) observa que "a capacidade competitiva de uma empresa está intimamente relacionada à conjugação de gestão e informação tecnológica, devido às crescentes exigências do mercado com relação a novos produtos e serviços de alto conteúdo tecnológico".

Quando uma empresa decide alterar sua estrutura visando reduzir sua verticalidade, os velhos sistemas de informação implantados, sofrem grande impacto porque não retratam mais a realidade e as novas necessidades de informação. Se não forem remodelados, podem prejudicar e até inviabilizar a mudança estrutural. Se forem vistos como importante ferramenta impulsionadora do processo de mudança, podem ser replanejados e aperfeiçoados, ou totalmente alterados, de acordo com os novos paradigmas da organização (SPINOLA; PESSOA, 1997)

Neste contexto, a informação, sob o impacto da utilização da tecnologia da informação, tem uma influência cada vez maior na organização do futuro. A introdução de novas tecnologias de informação nas organizações ampliou as potencialidades da informação como recurso estratégico, a velocidade com que a interação entre gestão e informação ocorre e a qualidade desta ligação. Estes avanços tecnológicos modificam as relações entre tempo e espaço. As distâncias temporais e espaciais cobertas pelas novas tecnologias tornam o passo de vida cada vez mais rápido:

\footnotetext{
"Uma organização só consegue ser inteligente, bem informada e sábia se o seu pessoal for sábio e estiver interligado. Nenhum padrão fixo de interligação servirá. A combinação correta de mentes muda com a rapidez com que a organização passa do exame de antigos problemas para os novos. Cada mudança requer o aprendizado de novos padrões e de novas competências" (PINCHOT, 1994).
}

As tecnologias de informação, por serem responsáveis pela captação, armazenamento, tratamento e disseminação da informação, têm sido utilizadas intensamente em empreendimentos onde o recurso informação/conhecimento é de grande relevância, como no caso das organizações ou áreas dentro das organizações cujo negócio é a pesquisa e desenvolvimento.

\section{Tecnologia da informação e a gestão de RH}

Um sistema baseado em computador quase sempre tem um elemento humano, que pode interagir diretamente com o hardware e com o software, realizando um diálogo que direciona a função do sistema. (PRESSMAN, 1995).

Santos (1998, p. 91-92) apresenta as seguintes afirmações como nova postura estratégica de gestão de recursos humanos voltados para a criação de novas vantagens competitivas para os negócios: 
- $\quad$ "As estratégias de recursos humanos envolvem uma tentativa de estabelecer uma relação direta entre prioridades estratégicas e os processos organizacionais que produzirão os comportamentos necessários para a sua consecução. Portanto, as estratégias de recursos humanos devem estar integradas com a estratégia empresarial no sentido de serem mutuamente apoiadoras";

- "Conseguir vantagem competitiva por meio das pessoas envolve, fundamentalmente, a alteração de nossa maneira de pensar sobre a força de trabalho e a relação empregatícia. Significa conseguir êxito trabalhando com pessoas, não substituindo-as ou limitando o escopo de suas atividades. Vincula ver a força de trabalho como fonte de vantagem estratégica, não apenas como custo a ser minimizado ou evitado";

- $\quad$ "A área de recursos humanos deve tomar a iniciativa de identificar as questões relacionadas às pessoas e lutar por sua consideração no planejamento de negócios corporativos" (BROWN).

Meshoulam e Baird (1987), apontam que a empresa deve desenvolver uma nova perspectiva sobre o gerenciamento de recursos humanos. Essa nova perspectiva deve explicar como as organizações estão mudando e como os recursos humanos podem proativamente antecipar e responder às suas necessidades.

Chang Júnior (2001) mostra a importância do comprometimento dos empregados com os objetivos organizacionais que deve estar evidenciado em função do novo paradigma de produção industrial: a automação industrial. Coloca ainda que nas novas técnicas emergentes da automação integrada flexível, que tem na diversificação um fator de vantagem competitiva e de captura de novos mercados, os recursos humanos ao invés de alienados e despreparados, exigem um quadro de empregados qualificados e comprometidos para participarem ativamente do processo produtivo. Aponta ainda, três fatores que auxiliam o no funcionamento das organizações:

- As pessoas devem ser induzidas a entrar e a permanecer no sistema;

- Devem desempenhar seus papéis específicos de maneira confiável;

- Ser inovadoras e atuar espontaneamente, além das prescrições do seu papel.

Abreu e Resende (2001) apresentam algumas habilidades do profissional que estará implantando uma TI:

- Habilidades técnicas;

- Habilidades de negócios;

- Habilidades comportamentais.

Essas habilidades são exigidas para todos aqueles que estão envolvidos no processo de implantação da TI independente do nível de atuação, de maneira a disseminá-la e distribuí-la por toda a empresa. 


\section{Comportamentos funcionais das pessoas para implantação de TI}

Ao adotar a implantação de uma TI, as empresas têm buscado, no perfil dos seus funcionários, as seguintes características:

- Alto grau de comportamento criativo;

- Focado nos projetos de longo prazo;

- Alto grau de cooperação e comportamento interdependente;

- Nível de preocupação equivalente para processos e resultados;

- Alta tendência para assumir riscos;

- Alta tolerância para com a ambigüidade e imprevisibilidade.

As empresas que implantam tecnologias de informação tendem a ter as seguintes implicações:

- Seleção de pessoal altamente qualificado;

- Altos investimentos em RH;

- Controles menos rígidos das pessoas;

- Mais recursos para experimentações;

- Recompensa de eventuais falhas e avaliação da performance com base nas ações de longo prazo.

Assim a adoção de uma estratégia de TI representa grandes implicações para o gerenciamento dos recursos humanos. Mais do que enfatizar que se controle as pessoas para que trabalhem mais ou melhor em cima dos mesmos produtos ou serviços, a estratégia para implantação de uma TI requer que as pessoas trabalhem diferentemente.

\section{Práticas de RH associadas a implantação de uma TI}

Baseados nos comportamentos funcionais apontados no item 4, as empresas que implantam uma TI tendem a possuir as seguintes características:

- Maior interação e coordenação entre equipes de trabalho;

- Avaliação de performance focada no longo prazo e metas por equipes;

- Empregos que possibilitam aos funcionários desenvolver habilidades que podem ser utilizadas em outras áreas da firma;

- Sistemas de compensação que enfatizam igualdade interna mais do que igualdade com o ambiente externo ou com o mercado; 
- Propiciar aos funcionários participação na empresa através da aquisição de ações ou a liberdade para que escolham a combinação de benefícios (salário, bônus, bens) que formem seus pacotes de rendimentos;

- Amplas opções de carreiras que reforce uma ampla gama de habilidades. Estas práticas facilitam a cooperação, o comportamento interdependente que é orientado ao longo prazo e estimulam a troca de idéias e a tomada de riscos.

\section{Fatores que auxiliam a estratégia de RH na implantação de TI}

Dutra (2002) apresenta um modelo de gestão de pessoas que pode ser adaptado para auxiliar na implantação de estratégia de RH. Essse modelo apresenta as seguintes idéias:

- Desenvolvimento mútuo: a gestão de pessoas deve estimular e criar as condições necessárias para que empresa e pessoas possam desenvolver-se mutuamente nas relações que estabelecem. Esse desenvolvimento significa o processo que permite à empresa e às pessoas atuarem em realidades cada vez mais complexas e exigentes;

- Satisfação mútua: a gestão de pessoas deve se alinhar como os objetivos estratégicos do negócio da organização e o projeto profissional e de vida das pessoas;

- Consistência no tempo: a gestão de pessoas deve, também, oferecer parâmetros estáveis no tempo para que, dentro de uma realidade cada vez mais turbulenta, seja possível à empresa e às pessoas terem referenciais para se posicionarem de forma relativa em diferentes contextos e momentos dessa realidade.

As seguintes condições, apresentadas por Dutra (2002), são utilizadas para assegurar efetivação de um modelo de gestão que podemos utilizar na implantação de TI:

- Transparência: a clareza dos critérios que norteiam a gestão de pessoas e a contínua transmissão de informações sobre as estratégias do negócio da empresa é fundamental para o sucesso;

- Simplicidade: a simplicidade na formulação dos critérios e na forma de sua aplicação ajuda na transparência e é fundamental para facilitar a compreensão, a análise, a discussão, o consenso, a aceitação e o comprometimento em torno dos valores e de sua prática em cada contexto específico;

- Flexibilidade: os critérios que norteiam a gestão de pessoas são validados a cada momento ao longo do tempo e permanecem se tiverem a flexibilidade necessária para ajustar-se aos diferentes contextos existentes e às pressões impostas pelas transformações desses contextos no tempo.

Para apoiar a construção do modelo de gestão de pessoas, DUTRA (2002) apresenta as seguintes premissas conceituais que estão focadas no: 
- Desenvolvimento em vez de foco no controle;

- Processo em vez de foco nos instrumentos;

- Interesse conciliado em vez de foco no interesse da empresa;

- Modelo integrado e estratégico, em vez de foco no modelo constituído por partes desarticuladas entre si.

\section{Considerações finais}

Muitos profissionais e gerentes reconhecem a necessidade de práticas de gestão de recursos humanos baseadas nos negócios da empresa. O aumento da complexidade, mudanças nas tecnologias, altos níveis de competição e a necessidade por flexibilidade fazem das pessoas um recurso ainda mais importante.

A base industrial da economia está se deslocando para o setor de serviços e de informações. Métodos de produção e de trabalho estão mudando drasticamente.

A tecnologia da informação tem sido utilizada como ferramenta para melhorar os negócios da empresa e aumentar a eficácia da organização.

As organizações estão mudando e precisam de práticas de recursos humanos afinadas unicamente às suas necessidades atuais emergentes. Não é suficiente simplesmente melhorar ou apurar as práticas e programas utilizados, é necessária uma gestão de recursos humanos totalmente diferente, baseada no entendimento da organização agora e no futuro.

$\mathrm{Na}$ medida em que as organizações crescem e se desenvolvem, suas necessidades de recursos humanos se modificam e, para que sejam eficientes, é necessário que cresçam junto com a organização. Simultaneamente, os componentes internos dos programas de gestão de recursos humanos, os sistemas e as práticas precisam ser eficientemente organizados para que forneçam suporte uns aos outros.

A empresa precisa desenvolver um modelo estratégico para a área de recursos humanos, discutindo sua atuação na implantação de TIs nos sistemas produtivos.

Apontamos a seguir questões que consideramos importantes para poder associar a implantação de uma TI à estratégia de RH e que, se não forem bem solucionadas, podem pôr em risco o investimento feito:

- Quais as Estratégias de Recursos Humanos para atender a implantação de uma TI;

- Quais impactos serão gerados na organização e nas pessoas;

- Identificar os fatores de sucesso que possam fornecer suporte à estratégia da área de RH e integrá-la a estratégia de negócios da empresa; 
- Medir a reação causada pela disseminação da informação e conseqüente perda de poder pela gerência com a implantação da TI, assim como se deu o compartilhamento da informação;

- Determinar quais vantagens competitivas foram adquiridas pela implantação da TI e da estratégia de recursos humanos adotada;

- Perceber quais problemas gerados e soluções adotadas pelo RH;

- Quais características facilitadoras e limitadoras de RH surgiram na implantação da TI.

A adoção da Tecnologia de Informação induzirá novas formas de administração e necessariamente, um novo tipo de profissional de gestão, com características diferentes das adotadas em organizações mais tradicionais. Com isso um novo modelo de Gestão de Recursos Humanos deverá ser implantado para fazer frente a esse novo desafio.

Assim, "O foco da administração de recursos humanos necessita ser ampliado para um foco voltado para o contexto organizacional, e o uso inteligente dessas variáveis para apoio às estratégias de negócio. Esta compreensão reforça a idéia de que administração de recursos humanos é a administração da cultura. Porque todas as funções de recursos humanos, integralmente, devem expressar os valores dominantes da cultura que sejam profícuos para o desenvolvimento das organizações". (HANASHIRO, in SANTOS, 1980).

\begin{abstract}
The article presents a bibliographic review and analyses on the process of the integrating the implementation of information technology applicable to operations management with strategies and practices of human resources management. The use of information technology leads to new ways of management, demands a new profile of management expert with different characteristics from the ones currently adopted in companies, as it implies the development of new models of human resource management as well.
\end{abstract}

Key words: Information technology; business management; human resource management

\title{
Referências
}

ALCÂNTARA, R. C.; SILVA, A. L. Relacionamentos e estratégias para melhor coordenação da cadeia de suprimentos, Revista de Administração, São Paulo, v. 36, n. 3, p.49-58, jul./set. 2001.

ABREU, A. F.; REZENDE, D. A. Tecnologia da informação: aplicada a sistemas de informações empresariais, 2. ed. São Paulo: Atlas, 2001.

CHANG JÚNIOR, J. Gestão de pessoas pelo desenvolvimento do comprometimento organizacional: uma abordagem holística e simultânea dos determinantes envolvidos no processo. Tese (Doutorado), Universidade de São Paulo, Faculdade de Economia e Administração e Contabilidade. São Paulo, 2001.

DRUCKER, P. Sociedade pós-capitalista. São Paulo: Pioneira, 1995.

DUTRA, J. S. Gestão de pessoas: modelo, processos, tendências e perspectivas. São Paulo: Atlas, 2002.

FERREIRA, J. R. Informação é instrumento essencial para a competitividade na indústria. TECBAHIA, Camaçari, v. 9, n. 3, p. 5-6, 1994. 
FURTADO, J. S. Informações para a empresa. In: CONTADOR, J. C. (Coord.). Gestão de operações. São Paulo: Edgar Blücher, 1997.

LAUDON, K. C.; LAUDON, J. P. Management infomation systems: new approaches to organization \& technology. New Jersey: Prenth Hall, 1998.

LAURINDO, F. J.B. Estudo sobre o impacto da estruturação da tecnologia da informação na organização e administração das empresas. Dissertação (Mestrado), Universidade de São Paulo, Escola Politécnica, Departamento de Engenharia de Produção. São Paulo, 1995.

. Um estudo sobre a avaliação da eficácia da tecnologia de informação nas organizações. Tese (Doutorado), Universidade de São Paulo, Escola Politécnica, Departamento de Engenharia de Produção. São Paulo, 2000.

MARTIN, E. W.; DEHAYES, D. W.; HOLFTER, J. A.; PERKINS, W. C. Management information technology: what managers need to know. New Jersey: Macmilian Publishing Campany, 1994.

MESHOULAM, I.; BAIRD, L. Proactive human resource manangement. Human Resource Manangement, v. 26, n. 4, p. 483-502, 1987.

cross ${ }^{\text {ref }}$

PINCHOT, G.; PINCHOT, E. O poder das pessoas. Rio de Janeiro: Campus, 1994.

PRESSMAN, R. S. Engenharia de software. São Paulo: Makron, 1995.

SANTOS, F. C. A. Dimensões competitivas da estratégia de recursos humanos: importância para a gestão de negócios e empresas manufatureiras. Tese (Doutorado), Escola de Administração de Empresas da Fundação Getúlio Vargas. São Paulo, 1998.

SPINOLA, M. M.; PESSOA, M. S. P. Tecnologia da informação. In: CONTADOR, J. C. (Coord.). Gestão de operações. São Paulo: Edgar Blücher, 1997.

WALTON, R. E. Tecnologia da informação: o uso da TI pelas empresas que obtêm vantagem competitiva. São Paulo: Atlas, 1993. 